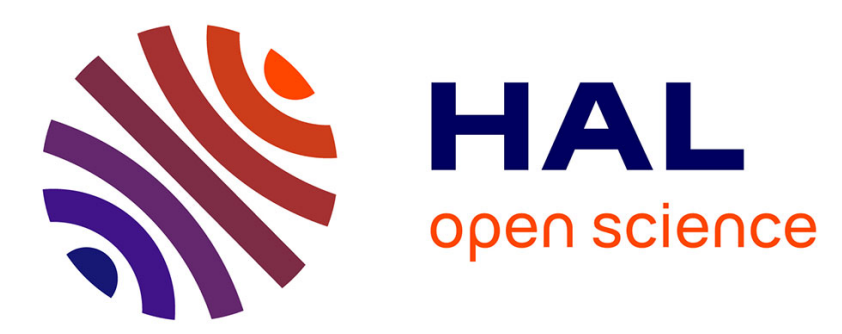

\title{
Pour une politique sociale européenne: les espoirs et les déceptions de Raymond Rifflet à la direction générale des Affaires sociales (1970-5)
}

Bertrand Vayssière

\section{- To cite this version:}

Bertrand Vayssière. Pour une politique sociale européenne: les espoirs et les déceptions de Raymond Rifflet à la direction générale des Affaires sociales (1970-5). European Review of History / Revue européenne d'histoire, 2018, 26 (2), pp.284-304. 10.1080/13507486.2018.1479376 . hal-01889330

\section{HAL Id: hal-01889330 \\ https://hal.science/hal-01889330}

Submitted on 18 Mar 2019

HAL is a multi-disciplinary open access archive for the deposit and dissemination of scientific research documents, whether they are published or not. The documents may come from teaching and research institutions in France or abroad, or from public or private research centers.
L'archive ouverte pluridisciplinaire HAL, est destinée au dépôt et à la diffusion de documents scientifiques de niveau recherche, publiés ou non, émanant des établissements d'enseignement et de recherche français ou étrangers, des laboratoires publics ou privés. 


\title{
Pour une politique sociale européenne : les espoirs et les déceptions de Raymond Rifflet à la direction générale des Affaires sociales (1970-1975).
}

\author{
Bertrand VAYSSIÈRE, Maître de conférences à l'Université Toulouse 2-Jean-Jaurès.
}

RÉSUMÉ : La fin des années 1960 voit une évolution dans la politique sociale européenne, impulsée par Bruxelles et avec le soutien apparent des États : réforme du FSE, Comité permanent de l'emploi, tout semble mener d'une simple action subsidiaire à un projet ambitieux de prise en compte des forces sociales dans le processus communautaire. Ce revirement, aujourd'hui oublié car ayant échoué, a eu ses témoins et ses acteurs, qui ont pu impulser les réformes qu'ils pensaient adéquates, et cru un temps en leur succès. Parmi ceuxci, nous allons étudier Raymond Rifflet, alors directeur général aux Affaires sociales, fervent défenseur d'une Europe fédérale proche de ses peuples, qui a joué un rôle important dans une période d'intense activité, bientôt stoppée par la crise.

Militant socialiste et fédéraliste belge avant de devenir chef de cabinet du Président de la Commission européenne Jean Rey en 1967, Raymond Rifflet fait partie de ces hommes qui soutiennent une activité plus intense de la CEE dans le secteur social. Très actif au Mouvement fédéraliste européen (MFE) dont il finit par être le président, il s'est engagé dans divers réseaux européistes et socialistes, où il a toujours plaidé dans ce sens ${ }^{1}$. Mais cet homme de convictions connait une impulsion décisive dans sa jeune carrière de fonctionnaire européen en intégrant, en juillet 1970, la direction générale des Affaires sociales (DG V), passant sous l'autorité du commissaire en charge de ce dossier, Albert Coppé. Le contexte semble bon : la question sociale tient à la fin des années 1960 une place grandissante dans l'agenda de la Commission européenne, alors que la croissance économique peine sur sa lancée et semble même se ralentir. Certes, une politique sociale européenne s'est bien dessinée en filigrane au sein de la CEE, mais celle-ci ne repose pas sur une formule endogène : dans une période de forte croissance, les Européens s'en remettent aux forces du marché pour l'amélioration des conditions sociales, tout en comptant sur l'approche interventionniste et redistributrice que continuent à privilégier et à assurer les différents États membres. Ce «mélange vertueux de dirigisme national et de libéralisation européenne »2, dont les origines peuvent être trouvées dans les débats menées dès les années 30 au sein du Bureau international du Travail, ne nécessite pas de dispositions sociales particulières de la part de la Commission jusqu'à la fin des années 60. De fait, celle-ci limite son action à des secteurs pointus tels que la sécurité au travail et la formation professionnelle, dans lesquels son intervention ne s'appuie que sur des mesures incitatives de la part du très modeste Fonds social européen (FSE). À cette époque cependant, les premiers nuages s'accumulent sur la croissance, jusqu'alors pivot de l'Europe, ce qui entraîne au sein de la Commission les premières réflexions hardies sur une politique sociale européenne à part entière ${ }^{3}$.

Il est donc intéressant de voir quelles réflexions et quelles propositions sont faites dans le cadre de cette apparente réorientation de l'action communautaire, entre le sommet de La Haye de 1969 et les premières années post-crise, en prenant comme fil directeur les actions de Raymond Rifflet, et en suivant trois séquences : celle des espérances, celle des initiatives, puis celle de la déception. Car en effet, en un temps très court, une vision sociale apte à renouveler la politique communautaire, portée par un contexte et un milieu en apparence favorables, s'éloigne, ne résistant pas aux turbulences initiées par la crise et l'élargissement européen. Quelles sont les raisons de cette fragilité ? Pourquoi le virage social, espéré par des hommes comme Rifflet, n'a-t-il pas pu être négocié ?

I- Un tournant annoncé dans la politique communautaire. 
1) Un changement de contexte.

La politique sociale européenne est abordée dans le préambule du Traité de Rome, les États membres s'assignant pour but l'amélioration des conditions de vie et d'emploi de leurs peuples, deux chapitres étant consacrés à la «politique sociale », que l'on ne dit cependant pas «communautaire » comme la PAC. Dans ce domaine, la Commission a le pouvoir de mener des études, des avis et des consultations, soit des mesures qui n'ont rien de contraignant, et qui doivent respecter deux lignes de force : assurer un haut niveau d'emploi en éliminant le chômage par la libre circulation et la formation professionnelle et en garantissant des droits analogues à tous les travailleurs qui se déplacent dans la Communauté (article 2 du Traité de Rome) ; améliorer les conditions de vie et de travail de la main-d'œuvre grâce à l'« égalisation dans le progrès » des systèmes sociaux (article 117). La logique de la CEE reste avant tout économique, à l'heure d'une production inédite de richesses dans chacun des pays qui en sont membres. La politique sociale, de ce fait, est fragmentaire, alors que le FSE, qui aurait pu s'appuyer sur un budget conséquent pour une action efficace, n'atteint un montant de dépenses que de 420 millions d'écus seulement pour toutes les années 1960. Les organisations syndicales se plaignent eux-mêmes très vite de son fonctionnement4, d'autant plus que leur rôle reste limité par les dispositions du Traité de Rome. Ils sont d'ailleurs conscients de cette faiblesse, surtout auprès du principal organe issu des Traités, le Conseil des ministres5. En 1958, un Secrétariat syndical européen a bien été institué, mais les syndicats restent marginalisés dans le processus de décision, et se sentent de plus en plus écartés alors que se profile la mise en place du Marché commun ${ }^{6}$.

Certes, une DG V «Affaires sociales » a bien été créée dès 1958 à la Commission, qui fait partie des neuf directions générales originelles, mais le traité CEE reste plus centré sur l'individu en tant qu'opérateur économique, concerné d'abord par la liberté de circulation (art. 48 à 51) et d'établissement (art. 52 et suivants). Dans cette optique, les DG I (Relations extérieures), VI (Agriculture) et VIII (Développement et Coopération), «championnes de la croissance », sont mieux placées à l'heure du Marché commun, ce qui nous rappelle que les directions générales disposent d'un personnel, d'une histoire et partant de pratiques différentes largement influencées par le contexte ${ }^{7}$.

Qui plus est, la Commission n'est même pas unanime sur la question plus générale de la politique sociale qu'il faudrait mener à l'échelle communautaire : y a-t-il seulement une quelconque volonté à Bruxelles dans ce domaine? L'ancien commissaire en charge des Affaires sociales Giuseppe Petrilli (1958-1960) portait lui-même un jugement très sceptique sur ses propres capacités d'intervention ${ }^{8}$. Raymond Rifflet, lorsqu'il sera nommé, abondera dans ce sens dans le bilan qu'il fait alors de l'action communautaire de la CEE en matière sociale, «à la fois très large et très vague ${ }^{9}$, « en aval » des autres objectifs de la Communauté d'abord centrés sur la politique douanière et sur l'agriculture suivant les impulsions données par les négociateurs ${ }^{10}$. Il considère avec ironie que la personnalité la plus marquante de la Commission des années 1960 a été Hans von der Groeben, commissaire à la Concurrence, précisément un des postes les plus influents pour lutter contre l'émergence d'un « modèle social européen ». Ce bilan sert bien évidemment une stratégie discursive propre à légitimer un tournant que Rifflet a intérêt à dessiner comme nécessaire (l'action sociale communautaire n'est pas si indigente qu'il le dit; quant à von der Groeben, c'est un ordolibéral plutôt qu'un libéral pur et dur), mais s'insère dans un contexte qui est effectivement troublé.

Mai 68, suivi de «l'automne chaud» italien du mois de septembre 1969, relance plus généralement le débat sur le dialogue social. Une remise en question s'est ainsi faite sentir dans chacun des pays de la CEE, reconsidérant le modèle social qui a jusqu'ici largement 
accompagné les Trente Glorieuses, et cette remise en question parvient jusqu'à Bruxelles : la délégation de la RFA, État où a eu lieu un changement politique autour de Willy Brandt en faveur de la social-démocratie, propose en particulier une relance de la politique sociale européenne, largement appuyée par le puissant syndicat $D G B$. Le chancelier allemand évoque même, «avec une vigueur inhabituelle », l'instauration, au niveau européen, de contrats de travail collectifs et de mesures d'harmonisation sociale, propositions qu'il présentera au sommet de La Haye de décembre $1969^{11}$.

Ce soutien au plus haut niveau explique la tenue de la première conférence tripartite sur les problèmes de l'emploi à l'échelle de la CEE. L'insatisfaction des partenaires sociaux est alors à son comble, ce dont tiennent compte certains hommes politiques de premier plan : Giacinto Bosco, ministre italien du Travail et de la Prévoyance sociale, et son homologue luxembourgeois Pierre Krier avaient déjà défendu, lors d'une session du Conseil des Affaires sociales du 5 juin 1967, le principe de rencontres permanentes entre employeurs, travailleurs, ministres et Commission, sans succès ; cette proposition a été cependant reprise par ce même Conseil deux ans plus tard (24-25 novembre 1969), qui propose la tenue d'une conférence pour discuter des modalités qu'une coopération entre partenaires sociaux pourrait prendre. Longtemps retardée par la France, qui estime qu'une institution nouvelle n'aurait pas sa place dans le dispositif communautaire, celle-ci se tient à Luxembourg à la fin du mois d'avril 1970, rassemblant des représentants des syndicats (la CGT et la CGIL en font partie, sous pression notamment de la France ${ }^{12}$ ), des organisations patronales, de la Commission et les ministres du Travail des Six, dont certains sont d'anciens syndicalistes sensibles aux questions de partenariat social (Carlo Donat Cattin, Pierre Krier, Bauke Roolvink, Louis Major qui préside lui-même la Conférence). La France cède face aux événements de 1968, particulièrement durs chez elle, et à la pression des partenaires sociaux qui font connaître leur position sur ce dossier de manière groupée en faveur de cette conférence luxembourgeoise ${ }^{13}$. Leur revendication est de développer une politique commune de l'emploi, avec la constitution d'un «Comité permanent», qui favoriserait une véritable politique de reconversion. Un retournement de conjoncture est en effet en train de se produire au sein de la Communauté, qui voit la croissance ralentir, le chômage augmenter (on compte 1,4 million de chômeurs dans la CEE en 1970, et 1,6 dès l'année suivante ${ }^{14}$ ) et l'inflation reprendre.

On peut donc voir que la relance du débat social communautaire accompagne l'arrivée de Raymond Rifflet à son nouveau poste, en juillet 1967. Il voit d'entrée dans celle-ci une bonne affaire pour la Commission, lui permettant de jouer un «rôle pilote fondamental [dans] la politique sociale [qui s'inscrit] dans la nouvelle période de l'intégration $»^{15}$ : pour lui, il s'agit de dépasser la simple procédure de coopération, forcément ponctuelle, et de «politiser » la question sociale, surtout dans l'optique de l'union économique et monétaire, discutée au même moment. Le positionnement de Rifflet rencontre alors celui de nombreux observateurs sur les risques de dessaisissement démocratique liés à l'évolution des Communautés européennes, où certains transferts de pouvoir sont opérés des autorités nationales sous contrôle parlementaire vers des centres de décision européen qui en sont dispensés, observateurs qui préconisent, pour éviter ce dessaisissement, une implication plus forte des acteurs sociaux et du Parlement européen dans les réformes communautaires ${ }^{16}$.

Dans ce contexte, la formule du Comité économique et social européen créé en 1957 semble définitivement dépassée (et, après tout, l'homme qui préside la Conférence de Luxembourg, Louis Major, en est lui-même un ancien président), alors que le Parlement européen soutient une réforme d'ampleur ${ }^{17}$. Tenant compte de différentes revendications, le Conseil des ministres des Affaires sociales, suite à la Conférence luxembourgeoise ${ }^{18}$, donne ainsi mandat au Coreper d'établir, avec la Commission et en consultation avec les partenaires sociaux, le schéma d'une organisation plus ambitieuse en matière de dialogue social. 


\section{2) Les ambitions affichées de la DG V.}

Lorsque Rifflet entre à la Commission européenne en 1967, il semble qu'il ait tourné la page de ses années militantes au sein du PSB, de la FGTB et du Mouvement fédéraliste européen, qui ont tous trois monopolisé son action depuis la Libération. Il a été de tous les combats européens : présent au Congrès de La Haye de 1948, défenseur acharné de la CED, il a représenté la FGTB lors des discussions qui ont suivi le sommet de Messine et s'est fait un chantre de l'antigaullisme en participant au mouvement des clubs, notamment le Club JeanMoulin. Un autre monde s'ouvre à lui une fois nommé chef de cabinet du Président Rey, celui des milieux communautaires, qu'il ne connaissait que peu jusque-là. Concernant cette accession, il doit certes beaucoup aux liens et aux réseaux politiques et militants qui lui ont permis de rencontrer des hommes comme Jean Rey ou Giuseppe Petrilli, dans lequel il retrouve la même sensibilité aux questions sociales européennes. Le contexte de la fin des années 60 a aussi son importance, qu'il faut avoir à l'esprit pour comprendre que Rifflet, même s'il passe dans un monde - celui de l'administration - plus gestionnaire, n'abandonne pas pour autant ses convictions, bien au contraire.

C'est ce qu'il va pouvoir démontrer lorsqu'il devient directeur général à la DG $\mathrm{V}$, sous l'autorité du commissaire Albert Coppé. Le 2 juillet 1970, Franco Maria Malfatti succède à Jean Rey à la présidence de la Commission. La nouvelle administration a d'entrée affiché ses priorités, qui tournent autour de sujets aussi politiques que les relations transatlantiques, l'élargissement et l'union monétaire. Cependant, les perspectives de Rifflet ne sont pas réduites pour autant, bien au contraire : une note du secrétaire général de la CEE Émile Noël à l'intention de Malfatti, le 22 juin $1970^{19}$, attire ainsi l'attention du nouveau Président sur d'autres objectifs, où figurent les questions sociales et les rapports avec les partenaires sociaux.

La promotion de Rifflet au poste de directeur général n'est pas un fait quelconque, tant ce poste représente un rouage important du système communautaire. Les directions générales représentent en effet le sommet de l'administration européenne, dont les responsables occupent le grade le plus haut de la fonction publique communautaire. Ceux-ci supervisent leurs secteurs d'activité et représentent la principale courroie de transmission des commissaires, étant eux-mêmes assistés d'un mini-cabinet et suppléés par des directeurs généraux adjoints. À l'intérieur de chaque direction générale de la Commission, les différents acteurs, internes et externes, nouent des liens qui leur offrent une certaine identité de vues, et le directeur général, dans cette optique, joue un indéniable rôle de dynamiseur, si tant est qu'il ait la confiance de son commissaire. On peut même parler d'une «culture des directeurs généraux $»^{20}$, qui facilite une certaine prise de conscience et une communauté d'objectifs, se renforçant à l'intérieur même de leurs secteurs de compétences.

Concernant la direction générale où Rifflet est amené à exercer son action, son passé militant et ses aspirations sociales deviennent un atout, dont Coppé a tenu compte au-delà des appartenances partisanes (il est lui-même démocrate-chrétien), en accord avec la culture particulière de ce segment de l'activité communautaire. Avec la DG V en effet, nous sommes bien dans une structure qui affiche de manière nette ses ambitions politiques, aidée en cela par un contexte en apparence favorable, ce qui contribue à lui donner une identité particulière qui la rend assez atypique. Alors que les autres directions générales privilégient une action où prévalent les approches juridiques et économiques, les acteurs de la DG V mettent en avant leur spécialisation académique propre : José Neirinck, premier directeur général de 1963 à 1967, avait ainsi soutenu une thèse en sciences sociales, alors que Rifflet a étudié l'histoire et a enseigné dans un Institut sociologique ; qui plus est, les deux hommes ont constamment publié des articles ou des essais spécialisés dans les domaines de la politique ou de l'Europe sociales $^{21}$. Ils ont donc un profil plus politisé, n'hésitant pas à afficher leurs opinions, qui 
proviennent de leurs engagements respectifs préalables à leur entrée à la Commission (Neirinck a été syndicaliste, Rifflet militant socialiste et européiste). Le profil de Rifflet paraît ainsi plus marqué que ceux d'autres directeurs généraux, suivant les critères que Didier Georgakakis a pu relever dans son analyse sociographique des fonctionnaires européens (certes pour une période plus contemporaine) $)^{22}$ : très spécialisé, tenant compte des compétences de l'intéressé et du fait qu'il exercera celles-ci dans le seul domaine social (contrairement à d'autres, qui ont un parcours beaucoup plus transversal), il est aussi plus engagé, puisqu'il s'appuie ouvertement sur des critères militants.

La décision des États de soutenir le Comité permanent de l'emploi évoqué lors de la conférence de Luxembourg est de toute manière un signe positif, qui semble démontrer que le dossier social prend de l'ampleur dans la nouvelle Commission (puisqu'elle est associée à cette création). Rifflet voit là l'occasion de professer ses idées au plus haut niveau communautaire ; de plus, Albert Coppé, qui est son supérieur à la CEE, apparaît comme un commissaire ambitieux, lui-même fin connaisseur des réalités européennes, puisqu'il est aux premiers postes depuis l'institution de la CECA. Son programme -dont Rifflet est l'un des rédacteurs- qu'il fait connaître dans un Mémorandum publié le 17 mars 1971 définit des objectifs généraux élevés, dans les domaines de l'éducation, de la protection sociale et bien sûr de l'emploi. Celui-ci s'intitule «Orientations préliminaires pour un programme de politique sociale communautaire ${ }^{23}$. Rifflet en écrit la dernière partie, « qui indique bien qu'il faut arriver [...] à une discussion au niveau communautaire pour les grandes orientations économiques et sociales comme on le fait dans chaque pays, parce qu'il était tout à fait illusoire de croire que l'on pourrait faire que l'opinion publique suive sans un dialogue social européen $[\ldots] »^{24}$. Ces Orientations ont pour objectif d'aboutir progressivement à un programme d'action cohérent, afin de réaliser peu à peu une étroite concertation des politiques sociales des États membres. Coppé évoque pour cela la nécessité de la concertation plutôt qu'une réglementation impérative, via l'engagement des partenaires sociaux dans le processus décisionnel de la Communauté, s'appuyant sur des objectifs (femmes, handicapés) qui reflètent les mutations radicales que vivent les sociétés européennes à cette époque.

La première note de Rifflet à son commissaire date du 10 juillet 1970, huit jours après avoir quitté sa fonction de chef de cabinet du Président, et elle porte sur le dossier brûlant de la réforme du $\mathrm{FSE}^{25}$. Cette réforme propose la division des ressources en deux parties : celle des interventions liées à des décisions spécifiques du Conseil, prises à la majorité qualifiée, et celles qui ne sont pas liées à une décision spécifique du Conseil, agréées par la Commission après consultation d'un Comité tripartite du FSE. Rifflet espère mener à bien, à partir de ce deuxième point, une forme de consultation permanente des partenaires sociaux ${ }^{26}$. Sur cette question, il est en accord avec Coppé, qui plaide de son côté pour que la Commission puisse ne pas se voir retirer la gestion du futur Comité permanent de l'emploi par le Conseil des ministres ${ }^{27}$, en accord également avec la Confédération européenne des syndicats libres qui réclame même, par la voix de la puissante $D G B$ et de sa représentante Maria Weber ${ }^{28}$, la mise sur pied d'un Centre d'études européennes sur la question de l'emploi.

La DG V peut ainsi faire connaître plus qu'elle ne l'a jamais fait auparavant son intention de contribuer au renouveau communautaire, comme elle l'expose, par le biais de Coppé, dans la « contribution à l'exposé de Monsieur le Président [Malfatti] concernant la prospective des affaires sociales communautaires », auquel Rifflet a mis la main :

Malgré un accroissement réel du produit national par personne occupée de plus de 60 p. 100 en dix ans, accroissement dî certainement, en partie au moins, aux impulsions apportées par la création du Marché commun, et malgré le travail considérable accompli dans le cadre des diverses Communautés créées par les traités de Paris et de Rome, il nous faut bien constater que l'intégration européenne n'est pas encore une réalité vécue au niveau de la vie quotidienne du plus grand nombre. Ceci est grave si l'on ne considère pas 
l'unification européenne seulement comme une 'bonne affaire' mais comme l'un des éléments essentiels de la construction d'une société nouvelle, non seulement plus efficace, mais aussi plus pacifique, plus fraternelle, plus humaine ${ }^{29}$.

Rifflet s'inscrit bien dans ce programme, évaluant les futures activités qui seront les siennes dans le cadre de ses nouvelles fonctions à Bruxelles : «Je crois que deux de nos tâches essentielles dans les prochaines années seront, d'une part, la création d'une véritable concertation avec les partenaires sociaux au niveau communautaire (c'est de cela que je m'occupe activement) et, d'autre part, une 'vitalisation' du Parlement européen actuellement plus décoratif qu'efficace $»^{30}$. Concernant le premier point, il est effectivement sur le pont dès les premières notes qu'il fait parvenir à Albert Coppé, recommandant à son commissaire la constitution d'un «bloc [...] de la Commission et des partenaires sociaux ${ }^{31}$ capable de s'imposer face au Conseil des ministres, la première jouant le rôle d' « éducateur », les seconds lui apportant leur appui politique ${ }^{32}$.

II- Des initiatives multiples et audacieuses.

1) Les Affaires sociales à la recherche d'une stratégie.

L'appel de Rifflet à la mise en place d'une véritable politique sociale européenne de la part de la Commission ne suffit pas en elle-même, tant l'ensemble des acteurs de l'institution bruxelloise doit tenir compte de la collégialité, dont ils constituent des rouages parmi d'autres ; on ne doit pas non plus oublier que le commissaire à la tête de la DG V, Albert Coppé, n'a pas la même culture politique que Raymond Rifflet, ce qui constitue un filtre indéniable aux idées les plus audacieuses que ce dernier serait tenté d'exprimer. L'action du directeur général doit de toute façon tenir compte du fait que les dossiers qu'il défend circulent entre les différents bureaux et les différents commissaires, avec le risque que la proposition de départ, qui passe par toute une série d'intermédiaires suivant la pratique du dialogue permanent, soit modifiée et amendée en bout de chaîne.

La vision qu'a Raymond Rifflet de ses nouvelles fonctions à la DG V est malgré tout ambitieuse : celles-ci sont partagées entre un champ théorique, aboutissant à la collecte et à la diffusion de l'information, et à un autre plus pratique et opérationnel lié à la gestion du FSE, qu'il espère dynamiser dans le cadre de la réforme, en cours, le concernant. Rifflet espère ainsi faire de la DG V la véritable inspiratrice d'une politique sociale disséminée dans différentes directions générales, notamment à la DG II (Affaires économiques et financières), où «les préoccupations sociales ne sont [pourtant] pas dominantes ${ }^{33}$. Au contraire, la DG V lui paraît être «la seule grande direction générale horizontale [qui veille] à ce que la dimension sociale de tous les problèmes soient traités en même temps que les autres, alors que les directions plus spécialisées ou verticales risquent d'être dominées par des considérations purement sectorielles sans voir les conséquences des mesures préparées sur la cohérence générale de l'activité communautaire ${ }^{34}$. Rifflet espère une dynamique plus forte, d'abord en rompant avec les habitudes de l'ancienne mouture, accusant, sur un ton volontairement provocateur, le commissaire aux Affaires sociales Lionello Levi Sandri, alors en poste (19601970), d'avoir abusé «du prétexte de son impuissance juridique » ${ }^{35}$. Dans le même temps, Rifflet souhaite que la DG V communique mieux avec les différents acteurs sociaux : c'est dans cette optique qu'est créé le bulletin Europe sociale, dans lequel sont amenés à s'exprimer des professionnels, soit de la Commission (Rifflet y rédigera plusieurs articles), soit des organisations professionnelles.

Fort de cette dynamique, Rifflet multiplie les prises de position, et se saisit à maintes reprises de son droit de proposition pour interpeller son commissaire. Partant de l'idée que la Commission doit être une boîte à idées, il n'hésite pas à suggérer des pistes novatrices, dans le 
but de permettre à l'institution bruxelloise d'être sur tous les fronts qui peuvent être ouverts dans le secteur de la politique sociale. Ainsi, Rifflet est-il l'un des premiers à proposer de mettre en chantier une réflexion sur une politique communautaire de protection de la santé et de l'environnement, souhaitant «profiter de l'émotion croissante qui se manifeste dans le monde à propos de la détérioration rapide de la qualité de la vie par la modification inquiétante de l'écologie et la multiplication des nuisances liées à une expansion industrielle purement quantitative $»^{36}$. Au même titre que le commissaire en charge des Affaires industrielles et de la Recherche -et ancien compagnon de lutte fédéraliste- Altiero Spinelli (1970-1976), Rifflet se montre ainsi sensible aux questions environnementales, tout en prônant une attitude pragmatique à propos de la communautarisation de ces dernières : cellesci doivent avoir pour but d'améliorer la qualité de la vie, mais également de lutter contre les distorsions de concurrence liées à des normes de protection qui ne sont pas les mêmes de nation à nation. Rifflet propose ainsi une collaboration dans le domaine environnemental avec la DG III de Spinelli, alors que le danger écologique vient d'être dénoncé par le Club de Rome et le rapport Meadows «Halte à la croissance ? ${ }^{37}$, inspirant certaines personnalités de la Commission, tel Sicco Mansholt. Cette proposition aboutit d'ailleurs à la création d'une division nouvelle consacrée spécifiquement à l'environnement au sein de cette DG III, sous la direction du Français Michel Carpentier ${ }^{38}$.

Le leitmotiv de Rifflet se retrouve dans cette ouverture aux problèmes les plus contemporains, et le pousse à garder un ton critique sur la façon dont la Commission les aborde : pour lui, la «période 'idéaliste' est, sinon passée, du moins devenue plus discrète. C'est déjà vrai au niveau des cadres supérieurs qui sont censés examiner les réalités du haut des pyramides... de papiers ; que dire, alors, des agents 'subordonnés' qui ne savent plus très bien à quoi ils le sont ? ${ }^{39}$. En dénonçant les lourdeurs du système, Rifflet ne fait que reprendre ici des thèses développées dans les clubs qu'il a fréquentés avant d'intégrer la Commission européenne (en particulier le Club Jean-Moulin), notamment celles de Michel Crozier sur le phénomène bureaucratique $^{40}$. Par ailleurs, il est conscient du manque de visibilité des fonctionnaires européens en particulier et des acteurs communautaires en général auprès des opinions. Rifflet est là aussi sur la même longueur d'onde qu'Altiero Spinelli, qui avait utilisé (le premier) le néologisme «eurocrate », non pas dans un but strictement polémique (comme c'est le cas aujourd'hui), mais dans une tentative de donner plus de sens au projet européen auprès du plus grand nombre ${ }^{41}$. Sa thèse, qui nous renseigne sur les succès et les limites de l'entreprise européenne au seuil des années 1970, est que l'Europe se fait bien, mais sans soubassement politique. C'est le triomphe du fonctionnalisme, qui déborde aussi bien les projets gaullistes que les espoirs fédéralistes en mettant en place un processus bureaucratique sans nature véritablement démocratique. Pour contrer cela, il s'agit pour la Commission de ne plus s'adresser aux mêmes cibles : plutôt que de viser en priorité des «centres de pouvoir non politiques » (administrations nationales, groupes d'intérêt), qui ne servent d'abord qu'à renforcer les pouvoirs de la Commission au mépris d'un vrai dialogue, celle-ci devra privilégier les contacts avec des «centres politiques » (partis, syndicats, Parlement européen, mouvements européistes), qui en retour lui donneront la légitimité dont elle manque cruellement.

2) Des réalisations d'envergure.

Il faut préciser que Rifflet peut compter sur l'appui d'autres membres de la Commission pour réclamer une systématisation du dialogue social à l'échelle européenne : c'est ainsi Jean Rey qui avait entamé dès février 1969 des négociations avec les syndicats les plus hostiles à la construction européenne, CGT et CGIL, démontrant une ouverture d'esprit rendue possible par l'évolution des relations internationales depuis les débuts de la Détente ; c'est Émile Noël 
qui milite pour que la Confédération européenne des syndicats participe au Comité permanent de 1 'emploi ${ }^{42}$. Cette ouverture est fortement soutenue par Rifflet, qui à plusieurs reprises a évoqué à demi-mot le peu de place que la Commission avait accordée jusqu'ici aux syndicats dans ses consultations, déjà à son poste de chef de cabinet du Président ${ }^{43}$, réclamant ce dialogue social qu'il avait toujours encouragé en tant que militant européiste.

Que donne l'état des lieux en la matière au début des années 1970 ? Les partenaires sociaux sont consultés de longue date dans le cadre communautaire, que ce soit au Comité consultatif de la CECA ou dans le Comité économique et social de la CEE; à partir des années 1960, des Comités consultatifs interprofessionnels tripartites assistent régulièrement la Commission (Comité du FSE, 1957 ; Comité consultatif pour la formation professionnelle, 1963) à propos de la libre circulation des travailleurs (1968) et de la sécurité sociale des travailleurs migrants (1971). On doit ajouter des Comités paritaires sectoriels qui sont réunis par la Commission à partir de 1963. Tous sont parties prenantes d'un dialogue à l'échelle européenne entre partenaires responsables et autonomes, mais tous n'ont cependant pas les ambitions du Comité permanent de l'emploi, dont l'idée a été actée après la Conférence de Luxembourg : il s'agit, avec ce dernier, de systématiser au niveau communautaire la concertation entre les institutions de Bruxelles et les représentants des employeurs et des travailleurs en vue de faciliter la coordination des politiques de l'emploi des États membres. Le Comité permanent permettrait ainsi des rencontres chaque semestre entre les partenaires sociaux et le Conseil, même si l'on ne doit pas oublier qu'il est avant tout à caractère consultatif.

Rifflet a conscience que cette instance doit tenir compte du rapport des forces dans la CEE, c'est-à-dire que la Commission n'aura pas le premier rôle au départ : le secrétariat du Comité permanent de l'emploi, une fois créé, est d'ailleurs confié au seul Conseil, ce qui est une manière d'avoir l'œil sur lui. Par ailleurs, Rifflet, proche des organisations syndicales, sait que ces derniers conservent une certaine défiance à l'égard de Bruxelles : il informe ainsi son commissaire que les partenaires sociaux sont mécontents de leurs contacts avec la DG V, avec laquelle ils entament des discussions à n'en plus finir qui ne donnent rien, frustrant jusqu'aux fonctionnaires européens eux-mêmes ${ }^{44}$. Rifflet sait très bien que les syndicats gardent une mémoire critique de la Commission des années 1960, qu'ils accusent de s'être plus occupée de croissance que de politique sociale. Celui-ci sait de plus que la formule du Comité permanent de l'emploi n'a pas le soutien de la Commission dans son ensemble, ce qui renvoie aux oppositions idéologiques qui existent en son sein, dont la majorité reste réticente à l'égard du dialogue social. Le Berlaymont est ainsi divisé sur le rôle qu'il doit jouer au sein de celuici, entre ceux qui réclament une place accrue (cabinets Mansholt - Agriculture, et Spinelli Politique industrielle et recherche) et ceux qui préconisent une simple tâche d'accompagnement (cabinets Barre - Économie et Finances - et Borschette - Concurrence), en attendant de l'exiger.

Rifflet n'est cependant pas isolé car, comme nous l'avons dit, le dialogue social est alors un thème porteur dans la plupart des pays occidentaux, alors que celui-ci est défendu par certains ténors de Bruxelles dont, Rifflet le reconnaît, son propre commissaire, Albert Coppé. Ce dernier a démontré qu'il était en phase avec les discussions qui ont entouré le Congrès de La Haye autour de l'activation d'une politique ambitieuse, dont il entend être, à son poste, un des acteurs ${ }^{45}$, s'appuyant notamment sur le point 12 du communiqué final par lequel «les chefs d'État ou de gouvernement reconnaissent l'opportunité d'une réforme du FSE, dans le cadre d'une concertation étroite des politiques sociales ». Pour Rifflet, «c'est là que l'on a obtenu [...] l'idée qu'il fallait approfondir la politique sociale (jusque-là, on avait toujours dit [qu'elle] devait rester quelque chose de limité, appartenant aux États, et où la CEE ne pouvait délivrer que des avis et des études). Le postulat était alors renversé : pas d'Europe sans union économique et monétaire, pas d'union économique et monétaire sans politique sociale ${ }^{46}$. 
Ce tournant espéré semble se confirmer avec la création du Comité permanent de l'emploi le 24 décembre $1970^{47}$, dont une tâche essentielle est d'assurer de façon continue, dans le respect des traités et des compétences communautaires, le dialogue, la concertation et la consultation entre le Conseil, les représentants des gouvernements des États membres (ministres du Travail), la Commission et les partenaires sociaux (18 sièges pour les syndicats et autant pour les organisations patronales). Les espérances de Rifflet sont comblées : les travaux de ce Comité auront pour principal objectif de faciliter la coordination des politiques de l'emploi des États membres en les harmonisant avec les objectifs communautaires. La DG V est ainsi légitimée par l'insertion d'un dialogue permanent dans l'agenda de la Commission ${ }^{48}$. L'idée que ce dialogue puisse englober social et économique est avalisée en instituant une discussion en continu, au sein de la nouvelle instance, avec tous les acteurs étatiques concernés, soit les ministres des Affaires sociales, mais également les ministres de l'Économie, au nom de la recherche d'efficacité maximale par intégration de tous les protagonistes dans le dialogue, notamment ceux qui sont censés détenir les cordons de la bourse.

Les perspectives semblent donc prometteuses, deux grands débats s'annonçant pour la décennie à venir : d'abord, quelle composante sociale pour l'union économique et monétaire, alors en pleine discussion? Dans le rapport Werner publié le 8 octobre 1970, il est exigé « la participation des divers groupes économiques et sociaux» pour réussir dans le marché commun « un développement satisfaisant, un haut niveau d'emploi, la stabilité, une réduction des disparités régionales et sociales, et la protection de l'environnement ${ }^{49}$; ensuite, comment réformer le FSE, dont il est prévu qu'il soit renouvelé le $1^{\mathrm{er}}$ mai 1972 , pour faire face aux défis qu'annoncent la nouvelle décennie ? Certes, tout le monde s'accorde pour que ses ressources soient augmentées, mais à un moment où les difficultés qu'il aura à gérer augmentent elles aussi.

Le FSE existe depuis l'origine des Communautés, mais sa dotation est limitée, et son fonctionnement est sujet à critiques, notamment de la part des organisations syndicales : effacer cette image médiocre représente un sérieux enjeu de crédibilité pour la Commission dans le cadre des négociations qui suivent la création du Comité permanent de l'emploi. Il s'agit donc d'opérer une réforme du FSE, elle-même programmée dans les traités, et c'est celle-ci que défend en bloc la DG V, notamment Rifflet qui y voit l'indispensable complément du dialogue social que la Commission souhaite mettre en place : il soutient ainsi les règles du Policy-mix à l'échelle communautaire, dont l'objectif est le plein emploi, que doit permettre d'atteindre la combinaison de la politique monétaire et de la politique budgétaire. Dans cette perspective, le FSE s'apparenterait à un fonds d'orientation sociale, pour que cette structure devienne un instrument de politique socio-économique entraînant un élargissement des perspectives communautaires.

C'est ainsi en apparence une véritable refonte du système, mais aussi de l'esprit du FSE, qu'entérine le Conseil des ministres le $1^{\text {er }}$ février 1971, annonçant une réforme effective du Fonds de manière à en faire l'instrument d'une politique plus active de l'emploi dans le cadre des nouveaux objectifs communautaires ${ }^{50}$. Le premier de ces objectifs est d'éviter la trop grande emprise des États membres et les dérives égoïstes qui ont caractérisé l'ancien FSE, sans oublier son manque criant de moyens, s'accentuant avec le temps et la montée des difficultés liées aux complications économiques croissantes. La conséquence en est la reconnaissance du rôle accru de la Commission dans le domaine social, puisque c'est elle qui hérite de la gestion du Fonds, indépendamment des administrations nationales. Le nouveau FSE, qui entre en vigueur le $1^{\text {er }}$ mai 1972, cofinance des actions définies «en fonction de critères communautaires et non plus nationaux » et décide « à l'avance de l'affectation de ses ressources au lieu de couvrir a posteriori les dépenses engagées par les États membres $»^{51}$. L'objectif est de transformer les modalités de financement pour faire de ce fonds européen un outil efficace en faveur de l'emploi, à destination de certains secteurs en difficulté et de 
certaines cibles. On peut considérer qu'on trouve là un « germe social » qui ne demande qu'à être développé. Trois domaines d'intervention nouveaux sont définis ${ }^{52}$ : dans le textile et l'agriculture et en direction des femmes de plus de 35 ans ; dans la réinsertion des handicapés au sein de l'activité économique, s'y ajoutant les travailleurs migrants ; dans la construction navale. Le FSE apparaît ainsi comme le catalyseur d'une politique systématique et moderne de l'emploi conçue dans le cadre communautaire, tirant des leçons de la solidarité économique à laquelle a conduit le développement du Marché commun.

\section{III- De l'espoir aux déceptions.}

1) Le sommet de Paris et ses suites.

Reste donc à officialiser ce tournant social pris par l'Europe au plus haut niveau, celui des conférences au sommet où peut se matérialiser une confluence entre ambitions communautaires et impulsions des États, nécessaires à la réalisation des premières. Le sommet de Paris du 19 au 21 octobre 1972 a lieu sous la houlette du président Georges Pompidou, et se présente comme un potentiel événement fondateur. En plus des six États membres, le Danemark, l'Irlande et le Royaume-Uni participent aussi aux discussions car leurs traités d'adhésion respectifs ont été signés. Trois thèmes sont prévus : union politique et renforcement institutionnel, relations extérieures de la Communauté, union économique et monétaire et progrès social.

Ce dernier dossier bénéficie de l'expertise apportée par le récent Plan Werner, et est particulièrement soutenu par la RFA, largement à l'initiative de l'agenda du sommet de Paris, avec pour base le programme sur lequel a été reconduit le deuxième cabinet Willy Brandt, qui définit le principe de la «démocratie sociale ${ }^{53}$. Le chancelier y présente un Mémorandum sur l'Europe sociale qui répond aux revendications adressées en juin 1972 par les syndicats européens aux chefs d'État et de gouvernement, notamment sur la création d'un institut européen du travail et la mise en place d'une formation professionnelle permanente ${ }^{54}$. Willy Brandt pèse sur les négociations, partant du constat qu'il $\mathrm{y}$ a, dans la politique communautaire, un déficit social (le point 3 du communiqué final du sommet, à la rédaction duquel il a plus particulièrement participé, refusera que l'expansion économique soit « une fin en soi $\gg^{55}$ ). Les participants reconnaissent que, pour la première fois depuis l'existence de la CEE, le niveau de vie de ses habitants est en passe de baisser, et que des solutions doivent être trouvées face à cette éventualité. Pour eux, «[...] une action vigoureuse dans le domaine social revêt la même importance que la réalisation de l'Union économique et monétaire. [Les États] invitent les institutions à arrêter, après consultation des partenaires sociaux, un programme d'action prévoyant les mesures concrètes et les moyens correspondants, notamment dans le cadre du Fonds social ${ }^{56}$. Le message est clairement adressé à la Commission, qui doit préparer ce programme avant le $1^{\mathrm{er}}$ janvier 1974, après consultation des partenaires sociaux; des dispositions sont également prévues concernant la politique régionale avec l'annonce de la création, «avant le 31 décembre 1973 », d'un fond de développement régional dont les aspects sociaux paraissent évidents, puisqu'il aura pour but « de corriger les déséquilibres régionaux dans la Communauté élargie, et notamment ceux résultant d'une prédominance agricole, des mutations industrielles et d'un sous-emploi structurel $»^{57}$.

À l'issue du sommet, les États s'engagent à «renforcer la Communauté en établissant une union économique et monétaire, gage de stabilité et de croissance, fondement de leur solidarité et base indispensable du progrès social, et en remédiant aux disparités régionales », le tout annonçant une Union européenne « avant la fin de l'actuelle décennie ${ }^{58}$. Ce résultat suffit au bonheur des membres de la Commission européenne qui recueillent là une « feuille 
de route $»^{59}$ encourageante, notamment Émile Noël qui a pu participer pour la première fois, en tant que secrétaire général de la CEE, au sommet des chefs d’État.

Pour répondre aux sollicitations de ces derniers, une cellule de réflexion est instituée dès le 25 octobre 1972, qui regroupe tous les directeurs généraux sous la présidence d'Émile Noël dans le but de travailler sur les domaines abordés et validés au cours du sommet de Paris : une première réunion de ce groupe baptisé «Suites du sommet » se tient le 6 novembre, avec pour objectif d'élaborer un inventaire des actions à entreprendre, d'autant plus vite que le collège des commissaires est amené à être renouvelé le $1^{\text {er }}$ janvier 1973. Raymond Rifflet fait naturellement partie de ce groupe ${ }^{60}$, et c'est lui qui rédige la partie consacrée à la politique sociale à mettre en œuvre à partir du sommet de Paris. Ce travail préparatoire est déposé le 8 janvier 1973 sur la table de la Commission ${ }^{61}$. Trois piliers le caractérisent: le premier concerne l'emploi, qui doit reposer sur une action communautaire systématique ; le deuxième s'intéresse aux conditions de vie et de travail, et évoque la défense de l'environnement; le troisième pilier a trait à la démocratisation des structures socio-économiques, notamment la question des instruments de concertation dans le cadre communautaire, pour laquelle le Comité permanent de l'emploi pourrait servir d'exemple.

C'est sur la base de ce rapport de la Commission que le Conseil adopte une résolution sur un programme d'action sociale le 21 janvier 1974 qui porte sur deux années et une quarantaine de mesures prioritaires, suivant trois objectifs principaux qui épousent ceux que soutient la DG V : la réalisation du plein emploi, l'amélioration des conditions de vie et de travail et la participation des partenaires sociaux aux décisions communautaires ${ }^{62}$. Ce programme met en place un cadre d'ensemble cohérent dans lequel s'insère le FSE à peine réformé. Celui-ci n'a plus seulement à intervenir après coup, sous forme de remboursements, mais peut devenir un acteur de la gestion de crise, notamment en matière de réinsertion de la main-d'œuvre en quête d'emploi, par la possibilité qu'il obtient d'orienter une partie de ses investissements, et donc de susciter des projets porteurs. Son action pourrait également s'exercer de manière préventive dans le secteur de la formation professionnelle. Les moyens accordés à ce fonds sont d'ailleurs nettement plus importants : entre 1958 et 1973, ils atteignaient 300 millions d'unités de compte (l'unité de compte est alors à peu près équivalente au dollar), soit 1,5 p. 100 du budget des Communautés européennes en moyenne. C'est peu, ce qui explique que les dépenses sociales sont d'abord assurées par la Haute Autorité de la $\mathrm{CECA}^{63}$; après la réforme (1974), ce pourcentage monte à 5,3 p. 100.

\section{2) Des limites.}

Dans les faits, cet enthousiasme doit être tempéré. N'oublions pas que la CEE reste toujours sous l'ombre portée du compromis de Luxembourg, le système intergouvernemental qu'il présuppose se renforçant bientôt avec la mise en place des Conseils européens ; il y a de plus de la part des États membres une certaine réticence à l'égard de toute action sociale ambitieuse à l'échelle communautaire, notamment la France pompidolienne, la plus en retrait sur la question des responsabilités européennes : le Président français, malgré ses bonnes dispositions apparentes au sommet de Paris, revient à des préceptes plus conservateurs une fois sa victoire assurée lors des législatives de mars $1973^{64}$. De manière plus générale, les chefs d'État et de gouvernement ont privilégié lors de ce sommet l'union monétaire, vue comme un préalable à l'Europe sociale, même par un homme comme Willy Brandt ${ }^{65}$. Or, la crise émergente menace cette union, elle-même étant la colonne vertébrale de toutes les politiques structurelles, dans les secteurs sociaux, régionaux et agricoles qui sont censées s'y rattacher; celle-ci pèse également sur les besoins des États membres, qui ne sont plus en rapport avec ce que peut leur proposer le nouveau FSE, même augmenté66 . 
Qui plus est, l'arrivée des Britanniques dans la CEE a une influence certaine sur le cours de la construction européenne, non seulement sur ses objectifs, mais également dans son organisation à travers le redéploiement des fonctionnaires que ces objectifs supposent. En effet, l'un des défis du début des années 1970 est d'intégrer les représentants des États désormais membres que sont le Royaume-Uni, le Danemark et l'Irlande, entraînant un véritable marchandage des portefeuilles à l'automne 1972, qui se résume à une terrible difficulté : «tenir compte des revendications nationales sans fragmenter à l'excès les domaines de compétence ${ }^{67}$. La négociation aboutit au plus haut niveau à la nomination de quatre commissaires ressortissants de ces nouveaux États membres : Christopher Soames aux Affaires extérieures, George Thomson à la Politique régionale, l'Irlandais Patrick Hillery aux Affaires sociales, et le Danois Finn Olav Gundelach au Marché intérieur. Cette réorganisation a tout de suite une incidence directe sur la carrière de Rifflet : après l'élargissement de 1973, il doit en effet laisser sa place de directeur général à un ressortissant britannique, Michael Shanks. La nomination de l'Irlandais Patrick Hillery à la tête des Affaires sociales n'a pourtant pas a priori de quoi inquiéter Rifflet : la politique sociale est incontestablement une préoccupation majeure pour son pays, l'un des plus pauvres de la CEE, et Hillery, en tant que ministre des Affaires extérieures, est celui qui a négocié le traité d'adhésion de ce dernier aux Communautés européennes ${ }^{68}$; c'est une question qui intéresse également le Royaume-Uni, en pleine reconversion industrielle, et qui place à la DG V, on l'a vu, un de ses ressortissants. De manière plus générale, le nouveau Président de la Commission François-Xavier Ortoli s'est engagé en faveur des objectifs arrêtés au Sommet de Paris, dont le FSE fait partie intégrante.

Raymond Rifflet n'a pourtant plus qu'un rôle secondaire dans la future action à mener : n'étant plus directeur général de plein exercice, il devient directeur général adjoint spécialement chargé des problèmes de l'emploi et du Fond social européen, toujours au sein de la DG V. Cependant, cette «promotion»n'en est pas une, puisque le représentant britannique, Michael Shanks, est le véritable patron administratif, ayant seul le titre de directeur général. L'interprétation de ce changement dans l'organigramme ne renseigne pas seulement sur le destin d'un homme, mais sur le changement de cap de toute une institution, pour des raisons internes (arrivée des Britanniques) et externes (installation dans la crise économique). Certes, Hillery, qui est désormais le destinataire des notes de Rifflet, est loin d'être un libéral, puisqu'il est affilié au Fianna Fail (centre gauche), mais il n'a aucune expérience, et pour cause, du système communautaire, dans le même temps où il ne soutient pas les aspirations fédéralistes exprimées par certains en son sein.

Malgré ces préventions, l'ancienne équipe de la DG V est en partie reconduite, la place de Rifflet n'étant pas pour l'instant remise en question, tout du moins en ce qui concerne une partie des dossiers dont il avait spécifiquement la charge, et d'abord le FSE ${ }^{69}$. Effectivement, ses activités sont désormais cantonnées à la gestion et à la défense de ce Fonds, dossier peutêtre trop technique pour un néophyte. Il est mobilisé pour présenter ses bilans tant qu'il est directeur général adjoint, mais aussi pour informer les nouveaux entrants sur cette ressource, ainsi que les régions dans leur ensemble, et enfin pour guider son commissaire dans les arcanes de la discussion autour du Fonds en Commission ou avec le Conseil, ou alors au cours des sommets européens, comme celui de Paris en décembre $1974^{70}$. Ce n'est pas pour autant une mise au placard, car les moyens à disposition du FSE ont effectivement augmenté de manière significative : plafonnés à 100 millions d'unités de compte dans le budget 1973, ils atteignent le chiffre de 550 millions trois ans plus $\operatorname{tard}^{71}$.

On distingue cependant une évolution qui confirme l'isolement croissant de Rifflet au sein de sa direction générale, cantonné à un rôle de gestionnaire qui n'a plus de prise avec l'action politique décidée de plus en plus en-dehors de la Commission. La plupart des notes qu'il adresse à son commissaire restent ainsi sans réponses, comme celle du 18 décembre 1973, dans laquelle il s'inquiète des effets de plus en plus visibles de la récession, et suggère une 
mobilisation du FSE pour aider les entreprises en difficulté ${ }^{72}$. À cette époque, la crise pétrolière a commencé et, même si le Conseil approuve le programme d'action sociale le 21 janvier $1974^{73}$, et qu'Hillery fait montre de sa bonne volonté dans toutes les enceintes où il s'exprime alors, le pessimisme l'emporte chez la plupart des observateurs, qui ne peuvent que constater une inversion des priorités communautaires que les difficultés économiques ont précipité $^{74}$. Rifflet n'est d'ailleurs pas dupe des décisions du sommet de Copenhague de décembre 1973, qui sont à ses yeux des «palabres » ${ }^{75}$, et dont le communiqué final « résultait moins d'une prise de conscience fédéraliste que de la peur des conséquences du désordre économique et monétaire ${ }^{76}$. L'ensemble lui paraît loin des témoignages de volonté politique donnés deux ans plus tôt à Paris, alors que la crise se profile de manière encore plus nette, remettant tout le système en question.

La conjoncture n'est pas seule en cause dans les difficultés qui pointent. Des problèmes plus structurels s'ajoutent, tournant autour de la différence de vision qui semble séparer les fonctionnaires communautaires de leurs homologues britanniques, qui les rejoignent alors à Bruxelles. Ceux-ci amènent avec eux une conception très rationnelle de l'action administrative, qui rompt avec une certaine tradition idéaliste qui pointait encore à l'époque de Jean Rey. On vit ici un peu le choc des cultures qui a heurté les Britanniques et les Continentaux à partir de l'élargissement. Au-delà des différences politiques, on peut parler de divergences stratégiques qui ont leur poids dans la gestion au quotidien. Le management, dont la pratique s'impose toujours plus à la Commission, fait incontestablement partie de cette conception, qui dérive sur la création en son sein d'un service "Effectifs, méthodes de gestion, évaluation ", dont l'objectif est d'améliorer le mode administratif propre à la Commission, alors qu'un premier manuel des procédures sera édité en mai 1976. Le nouveau secrétaire général adjoint Christopher Audland, nommé en mars 1973, veille en particulier à promouvoir une forme de "standardisation bureaucratique ${ }^{77}$ en rupture avec le système bruxellois antérieur privilégiant les contacts personnels et les vertus de l'expérience, qui après tout ont été des viatiques pour Rifflet ${ }^{78}$.

L'incertitude du contexte se reflète de plus en plus dans l'ordre de marche de l'agenda communautaire, des arbitrages étant rendus au sein même de la Commission qui renversent les perspectives précédentes : la remise des Orientations pour un programme d'action sociale que celle-ci devait faire parvenir au Conseil des ministres pour le mois d'avril 1973 est ainsi retardée à cause d'un manque de moyens et du contexte général qui en relativise la priorité ${ }^{79}$. Rifflet se rend compte de ce renversement de perspective, lui qui constate que sa direction générale est particulièrement touchée par les restrictions budgétaires que la Commission s'applique à elle-même, ramenant « [ses] effectifs à une limite inférieure à ce qu'ils étaient avant l'élargissement et avant qu'on ne parle de programme d'action sociale, avant aussi que le nouveau FSE ait pris les nouvelles responsabilités qui le caractérisent ${ }^{80}$.

L'attractivité de la DG V faiblit au sein même de la Commission, Rifflet se plaignant auprès de Michael Shanks que le principe du recours à la mutation volontaire ne présage « le départ d'excellents éléments qui préfèreront se diriger vers d'autres services que de se tuer à la tâche pour une politique que la Commission semble elle-même prendre si peu au sérieux ${ }^{81}$. La baisse des moyens accordés à ses services se confirme, Rifflet estimant dès 1974 la situation «intenable ${ }^{82}$. La répétition de ses courriers prouve que ses demandes restent sans réponse ${ }^{83}$. Par contre, ses préventions sur le départ des effectifs se confirme, le premier d'entre eux concernant Michael Shanks lui-même, parti en janvier 1976 pour devenir dirigeant d'entreprises privées au Royaume-Uni. La politique sociale communautaire est d'ores et déjà en déshérence lorsque Rifflet quitte la DG V au même moment, étant affecté à un poste, plus honorable qu'effectivement stratégique, de conseiller spécial du Président de la Commission Roy Jenkins en charge de l'information autour des futures élections européennes. Une page est tournée pour la politique sociale communautaire, alors que de plus en plus de personnes 
estiment que la meilleure relance de la CEE passe par l'institution d'un véritable marché unique...

\section{Conclusion.}

L'heure est bien au rétrécissement des choix communautaires, alors que les grandes options défendues par Bruxelles ne vont pas dans la même direction que celles imaginées par les défenseurs d'une politique sociale avancée, sans compter que les États gardent encore plus ouvertement l'initiative. Tout cela est la négation de ce que Rifflet avait espéré, lui qui, humiliation supplémentaire, doit quitter la DG V après avoir espéré que le départ de Shanks lui aurait permis de réintégrer son poste de directeur général de plein exercice. Si l'on avait pu avoir des doutes sur les raisons du déclassement de Rifflet en 1974, puisqu'il était en apparence justifié par les conséquences de l'élargissement et de la réorganisation des postes par nationalités qu'il suscitait, celles qui sont mises en avant pour faire barrage à la candidature du même homme deux ans plus tard ne souffrent plus d'ambiguïté : si être fédéraliste et proche des syndicats a pu aider Rifflet dans le choix qui l'a fait entrer dans la Commission européenne en 1967, ce n'est plus du tout un avantage dix ans plus tard, à l'heure où les pays membres de la CEE se sont bel et bien installés dans la crise. Certes, le syndicaliste belge Jean Degimbe prend la succession de Shanks à la DG V en 1976, mais il est à la tête d'une direction générale qui n'a plus la priorité dans l'agenda de la Commission.

Au-delà du cas personnel de Raymond Rifflet, c'est l'ensemble de la politique sociale européenne qui subit un coup d'arrêt, après les espoirs et les résultats concrets obtenus au début des années 70 (nouvelle pratique des conférences tripartites communautaires, augmentation des ressources du FSE, lancement d'un programme d'action sociale). Cet espoir a été partagé par les organisations syndicales elles-mêmes, qui créent en 1973 la Confédération européenne des syndicats, avec comme ambition de représenter les travailleurs à une échelle inédite. La crise a eu pour résultat de casser cette mécanique, et de ne faire de la Commission qu'un timide acteur aux réponses ponctuelles et d'ampleur modeste (création du Cedefop, nouvelle augmentation du FSE).

Ainsi, la construction européenne ne s'est jamais dégagée de la voie libérale qui a été la sienne depuis le départ. La différence, c'est que celle-ci paraissait acceptable dans les années 60 parce que la croissance était un amortisseur et que les États n'avaient pas abdiqué leurs fonctions régulatrices. À partir du milieu des années 70, sous l'effet de la crise, la priorité est donnée à l'approfondissement économique et à l'union monétaire, dont les conséquences entraînent un affaiblissement des États-providences et un engourdissement progressif de la politique sociale européenne qui a eu un temps -trop bref- voix au chapitre. Certes, un dialogue social européen est institué au milieu des années $80^{84}$, et certains textes communautaires proclament à l'envie une politique sociale européenne, au premier chef la Charte des droits sociaux de 1989. Cependant, l'intégration européenne, qui présuppose l'abandon des politiques nationales de soutien à la croissance, n'a pour l'instant pas proposé de solution équivalente à l'échelle plus large de l'Europe, montrant l'absence cruelle à ce niveau de la notion même d'intervention publique, alors que la crise est toujours là... 


\section{Notes}

${ }^{1}$ Sur la vie et les idées du personnage, nous renvoyons à Vayssière, Européiste et eurocrate : la vie fédéraliste de Raymond Rifflet.

${ }^{2}$ Mechi, « Du Bureau international... », 27.

${ }^{3}$ Varsori, “Alle origini di un modello sociale europeo...", 17-47.

${ }^{4}$ CESL, « Mémorandum sur le FSE », 29 octobre 1969, cité par Guasconi, "The Origins of The European Social Policy...", dans Varsori (ed.), 302.

${ }^{5}$ Schirmann, «Les syndicats ouvriers français et les institutions européennes », dans Bitsch, 289-290.

${ }^{6}$ Guasconi, « The Origins of the European Social Policy... », 301-311.

${ }^{7}$ Shore, 123-146.

${ }^{8}$ Cité dans Becherucci, « Lionello Levi Sandri au service de l'Europe », 122.

${ }^{9}$ Archives Historiques de l'Union européenne de Florence (désormais AHUE), Fonds Rifflet, Note à Monsieur Coppé, RR 1-2, 9 septembre 1970.

${ }^{10}$ AHUE, Fonds Mouvement européen, Raymond Rifflet, «La politique sociale dans la Communauté », ME2430, rapport sans date mais vraisemblablement de 1972.

${ }^{11}$ Olivi, L'Europe difficile, 159 ; Schirmann, «Willy Brandt et les débuts de l'Europe sociale », dans Wilkens (dir.), 311-323.

${ }^{12}$ La Commission avait également anticipé ce point, au nom de l'attitude plus constructive de l'URSS à l'égard de la CEE et de la constitution d'un bureau de liaison CGT-CGIL à Bruxelles en 1965.

${ }^{13}$ Lettre adressée au Conseil par l'UNICE et les secrétaires européens de la CISL et de la CMT le 9 mai 1968 ; revendications de la CESL à La Haye les 23-25 avril 1969 réclamant la « démocratisation de la Communauté », cité dans Paulus, La création du Comité permanent de l'emploi, 52.

${ }^{14}$ Muet, 47.

${ }_{15}^{15}$ AHUE, Fonds Rifflet, Note à Monsieur Coppé, RR 1-2, 9 septembre 1970, 5.

${ }^{16}$ Voir Meynaud, et Sidjanski, 476; Buchmann, «La capacité décisionnelle du système communautaire européen », dans Gerbet et Pépy, La décision dans les Communautés européennes, 461 ; Janne, Projet de rapport pour le congrès extraordinaire de la FGTB, 1971, cité dans Paulus, La création du Comité permanent de l'emploi, 100.

${ }^{17}$ Résolution du 3 juillet 1968, Journal officiel des Communautés européennes, 19 juillet 1968, n ${ }^{\circ} \mathrm{C} 72 / 40$.

${ }^{18}$ Qui a rassemblé 30 représentants des travailleurs (parmi lesquels 14 CESL, 7 OE-CMT, 3 CGIL-CGT, 1 CFTC, 1 DAG) et 30 représentants des employeurs (20 UNICE-COCCEE, 6 COPA, 4 CEEP).

${ }^{19}$ Cité dans Bitsch, in Dumoulin (dir.), La Commission européenne 1958-1972, 144.

${ }^{20}$ Abélès, « De l'Europe politique en particulier et de l'anthropologie en général », 35-58 ; Bellier, « Une culture de la Commission européenne?» in Dubois et Dulong (dir.), La Question technocratique. 233-253; Georgakakis et de Lassalle, «Les directeurs généraux de la Commission européenne », 6-33.

${ }^{21}$ Neirinck, La politique sociale du Traité de Rome et EEC Applied Labour Economics, 1969 et Politique sociale européenne de la CEE, 1970 ; les articles de Rifflet sont pléthore, avant et après sa nomination, dans les Cahiers de sociologie.

${ }^{22}$ Georgakakis et de Lassalle, «L'européanisation du personnel politico-administratif européen », in Baisnée et Pasquier, L'Europe telle qu'elle se fait, 55-75.

${ }^{23}$ Commission des Communautés européennes, «Orientations préliminaires pour un programme de politique sociale communautaire », Supplément, Annexe au Bulletin des Communautés européennes, 4, 1971, 2/71, Bulletin des Communautés européennes.

${ }^{24}$ Entretien de Raymond Rifflet avec Thierry Monforti, Archives Historiques de l'Union européenne, Florence, p. 146 .

${ }^{25}$ AHUE, Fonds Rifflet, Raymond Rifflet, Note à l'attention de Monsieur Coppé, RR 1-1, 10 juillet 1970.

${ }^{26}$ AHUE, Fonds Rifflet, Note à Monsieur Coppé, RR 1-2, 8 septembre 1970, RR 1-2.

${ }^{27}$ Albert Coppé, Création d'un Comité européen de l'emploi et convocation périodique d'un Conseil ad hoc de l'emploi, mémorandum $\operatorname{COM(70)~1072,~} 25$ septembre 1970.

${ }^{28}$ Membre du Bundesvorstand des Deutschen Gewerkschaftsbundes, elle est à la Commission chef de la division «Main-d'œuvre féminine » et du service « Formation professionnelle ».

${ }^{29}$ AHUE, Fonds Rifflet, Albert Coppé, «contribution à l'exposé de Monsieur le Président concernant la prospective des affaires sociales communautaires », RR 1-2, 2 septembre 1970. La patte de Rifflet est évidente, puisque l'exposé de Coppé reprend presque mot pour mot des phrases qui figurent dans des notes qu'il lui a fait parvenir, notamment dans AHUE, Fonds Rifflet, Note à Monsieur Coppé, RR 1-2, $1^{\text {er }}$ septembre 1970, 5. 
${ }^{30}$ AHUE, Fonds Rifflet, Lettre de Raymond Rifflet à Étienne de la Vallée Poussin, RR-119-2, 20 juillet 1970.

${ }^{31}$ AHUE, Fonds Rifflet, Note à l'attention de Monsieur Coppé, RR 1-1, 10 juillet 1970.

${ }^{32}$ AHUE, Fonds Rifflet, Note à l'attention de Monsieur Coppé, RR 2-2, 10 mars 1971.

${ }^{33}$ AHUE, Fonds Rifflet, Lettre de Raymond Rifflet à Alexandre Marc, RR 27-2, 27 septembre 1970.

${ }^{34}$ AHUE, Fonds Rifflet, Lettre de Raymond Rifflet à Alexandre Marc, RR 27-2, 27 septembre 1970, 2.

${ }^{35}$ AHUE, Fonds Rifflet, Note à l'attention de Monsieur le Président, RR 24-1, 11 décembre 1969. Pour une vision plus objective de l'action de Levi Sandri, Mechi e Varsori (ed.), Lionello Levi Sandri e la politica sociale europea.

${ }^{36}$ AHUE, Fonds Rifflet, Note à l'attention de Monsieur F. Vinck, RR12-2, 15 décembre 1970.

${ }^{37}$ Halte à la croissance ? : Rapport sur les limites de la croissance, également connu sous le nom de rapport Meadows, est le titre en français d'une expertise demandée à une équipe du Massachussetts Institute of Technology par le Club de Rome en 1970 : Meadows, Meadows, Randers et Behrens, The Limits to Growth.

${ }_{38}$ Interview de Michel Carpentier par Arthe Van Laer, 22 octobre 2010, European Oral History, archives.eui.eu/en/files/transcript/16415.pdf, 3-4.

${ }^{39}$ AHUE, Fonds Rifflet, Lettre de Raymond Rifflet à Guy Moentack, RR 7-2, 21 octobre 1971.

${ }^{40}$ Crozier, Le phénomène bureaucratique.

${ }^{41}$ Spinelli, The Eurocrats.

${ }^{42}$ Cité dans Bossuat, Émile Noël, 145.

${ }^{43}$ AHUE, Fonds Rifflet, Note à Monsieur le Président, RR 24-2, 19 septembre 1969.

${ }_{4}^{44}$ AHUE, Fonds Rifflet, Note à Monsieur Coppé, RR 2-1, 5 mars 1971.

${ }^{45}$ AHUE, Fonds Rifflet, Albert Coppé, « Relance de la politique sociale », RR-12-2, 17 décembre 1970.

${ }^{46}$ Entretien de Raymond Rifflet avec Thierry Monforti, Archives Historiques de l'Union européenne, Florence, 131.

${ }^{47}$ Décision du Conseil 70/532/CEE, du 24 décembre 1970 (Journal Officiel L273, 17 décembre 1970).

${ }^{48}$ AHUE, Fonds Rifflet, Albert Coppé, «Perspectives de la politique sociale communautaire », RR 2-2, 15 juillet 1971

49 «Rapport au Conseil et à la Commission concernant la réalisation par étapes de l'Union économique et monétaire dans la Communauté », 8 octobre 1970, Supplément au Bulletin des Communautés européennes, 111970, 10.

${ }^{50}$ Décision du Conseil, 71/66/CEE, du $1^{\text {er }}$ février 1971, JOCE 1971, L 28.

${ }^{51}$ Communautés européennes, Le nouveau fonds social européen, Textes officiels, Bruxelles, 1972.

${ }^{52}$ Articles 4 et 5 de la décision du Conseil du $1^{\text {er }}$ février 1971.

${ }_{53}^{53}$ Miard-Delacroix, Willy Brandt, 311-323.

${ }^{54}$ Guasconi, « Les syndicats et la relance de la politique sociale européenne », 61-69.

${ }^{55}$ Bulletin des Communautés européennes, octobre 1972, $\mathrm{n}^{\circ}$ 10, Luxembourg, OPOCE, 1. Dans ses mémoires, Brandt écrit que «le progrès social ne devait pas être un simple appendice de la croissance économique », Brandt, Mémoires, 371.

${ }^{56}$ Bulletin des Communautés européennes, octobre 1972, $n^{\circ}$ 10, Luxembourg, OPOCE, 5.

${ }^{57}$ Bulletin des Communautés européennes, octobre 1972, $n^{\circ}$ 10, Luxembourg, OPOCE, 5.

${ }^{58}$ Bulletin des Communautés européennes, octobre 1972, $n^{\circ} 10$, Luxembourg, OPOCE, 8.

59 Interview d'Edmund Wellenstein par Étienne Deschamps, La Haye, 27 août 2009, Centre Virtuel de la Connaissance sur l'Europe, http://www.cvce.eu/histoire-orale/unit-content/-/unit/c48e85ef-ca5a-42f5-9ab9de1c962cf61d

${ }^{60}$ Bossuat, ¿̇mile Noël, 230.

${ }^{61}$ AHUE, Fonds Rifflet, Direction générale des Affaires sociales, « Résumé des principales actions antérieures ou en cours, en matière de politique sociale, à la lumière des orientations données par le communiqué de la conférence au sommet de Paris », RR 9-1, fin 1972; AHUE, Fonds Rifflet, Direction générale des Affaires sociales, «Principales actions spécifiques à entreprendre au cours de l'année 1973 dans le domaine social en fonction de la conférence au sommet de Paris », RR 9-2, fin 1972; AHUE, Fonds Rifflet, Direction générale des Affaires sociales, «Premières indications pour l'élaboration de programmes d'action conformes aux décisions du sommet de Paris », RR 9-2, fin 1972.

${ }^{62}$ Résolution du Conseil du 21 janvier 1974 concernant un programme d'action sociale, Journal officiel $n^{\circ} \mathrm{C}$ 013, 12 décembre 1974, 1-4.

${ }^{63}$ Celle-ci a dépensé 250 millions d'unités de compte entre 1954 et 1973 pour aider 500000 travailleurs de la sidérurgie et des charbonnages, 400 millions pour la réadaptation de 230000 travailleurs de la sidérurgie par la suite, et a engagé 1,2 milliards de prêts pour la création de 63000 emplois.

${ }^{64}$ Schirmann, «Willy Brandt et les débuts de l'Europe sociale », 322-323.

${ }^{65}$ Schirmann, «Willy Brandt et les débuts de l'Europe sociale », 322.

${ }^{66}$ Leboutte, Histoire économique et sociale de la construction européenne, 308-310 et 442-450. 
${ }^{67}$ Badel et Bussière, François-Xavier Ortoli, 124.

${ }^{68}$ Walsh, Patrick Hillery.

69 "Belgian in Hillery's cabinet", The Irish Times, 18 janvier 1973; "Rifflet to be deputy director", The Irish Times, 7 février 1973.

${ }^{70}$ AHUE, Fonds Rifflet, Note à l'attention de Monsieur Hillery, RR 31-3, 26 novembre 1974.

${ }^{71}$ Walsh, Patrick Hillery, 367-368.

${ }^{72}$ GEHEC, Fonds Rifflet, Note à l'attention de Monsieur Hillery, farde n¹12, 18 décembre 1973.

${ }^{73}$ « Résolution du Conseil concernant un programme d'action sociale », 21 janvier 1974, Journal officiel $n^{\circ} \mathrm{C}$ 013 du 12 février 1974, 1-4.

74 “Commission unlikely to pass Hillery's social programme", The Irish Times, 27 septembre 1973.

75 «Déclaration sur l'identité européenne », Bulletin des Communautés européennes, n²12, décembre 1973, Luxembourg, OPOCE, 127-130.

${ }^{76}$ Raymond Rifflet, «Schéma de l'exposé introductif de M. Raymond Rifflet », Mouvement européen, réunion du comité directeur à Bruxelles, 19 janvier 1974, farde nº114, GEHEC, Louvain-la-Neuve.

${ }^{77}$ Ponzano, cité par Ramírez-Pérez, «Secrétariat général et service juridique », dans Bussière (dir.), La Commission européenne 1973-1986, 107.

${ }^{78}$ Sur les motivations de Christopher Audland, voir de lui Right Place - Right Time.

${ }^{79}$ AHUE, Fonds Rifflet, Lettre de Raymond Rifflet à Ernest Glinne, Président du Conseil des ministres des Communautés européennes, RR 103-1, 5 avril 1973.

${ }^{80}$ AHUE, Fonds Rifflet, Note à l'attention de Monsieur Shanks, RR-107-3, 25 février 1975.

${ }^{81}$ AHUE, Fonds Rifflet, Note à l'attention de Monsieur Shanks, RR-107-3, 25 février 1975.

82 AHUE, Fonds Rifflet, Note à l'attention de Monsieur Hillery, RR 106-1, 13 décembre 1974 ; AHUE, Fonds Rifflet, Note à l'attention de Monsieur Shanks, RR 106-2, 16 octobre 1974.

${ }^{83}$ AHUE, Fonds Rifflet, Note à l'attention de Monsieur Shanks, RR 107-4, 14 janvier 1975 ; AHUE, Fonds Rifflet, Note à l'attention de Monsieur Hillery, RR 107-3, 4 mars 1975.

${ }^{84}$ Lapeyre, Le dialogue social européen.

\section{Bibliography}

Abélès, Marc, «De 1'Europe politique en particulier et de l'anthropologie en général », Cultures \& Conflits, $\mathrm{n}^{\circ} 28$ (1997): 33-58.

Audland, Christopher, Right Place - Right Time, Stanhope: The Memoir Club, 2004.

Badel, Laurence et Bussière, Éric, François-Xavier Ortoli. L'Europe, quel numéro de téléphone?, Paris: Descartes \& Cie, 2011.

Baisnée, Olivier et Pasquier, Romain, L'Europe telle qu'elle se fait. Européanisation et sociétés politiques nationales, Paris: Éditions du CNRS, 2007.

Becherucci, Andrea, "Lionello Levi Sandri au service de l'Europe », Revue d'Histoire de l'intégration européenne, vol. 14, $\mathrm{n}^{\circ} 2$, (January 2008): 121-144.

Bitsch, Marie-Thérèse, Le couple France-Allemagne et les institutions européennes. Une postérité pour le Plan Schuman, Bruxelles: Bruylant, 2001.

Bossuat, Gérard, Émile Noël, premier secrétaire général de la Commission européenne, Bruxelles: Bruylant, 2011.

Brandt, Willy, Mémoires, Paris, Albin Michel, 1990.

Bussière, Éric, La Commission européenne 1973-1986. Histoire et mémoires d'une institution, Luxembourg: OPUE, 2014.

Crozier, Michel, Le phénomène bureaucratique. Essai sur les tendances bureaucratiques des systèmes d'organisation modernes et sur leurs relations en France avec le système social et culturel, Paris: Seuil, 1963.

Dubois, Vincent et Dulong, Delphine, La Question technocratique. De l'invention d'une figure aux transformations de l'action publique, Strasbourg: PUS, 1999.

Dumoulin, Michel, La Commission européenne 1958-1972. Histoire et mémoires d'une institution, Luxembourg: OPOCE, 2007. 
Georgakakis, Didier et de Lassalle Marine, «Les directeurs généraux de la Commission européenne : premiers éléments d'une enquête prosopographique », Regards sociologiques, $\mathrm{n}^{\circ}$ 27-28, 2004: 6-33

Gerbet, Pierre et Pépy, Daniel, La décision dans les Communautés européennes, Bruxelles: ULB, 1969.

Guasconi, Maria Eleonora, «Les syndicats et la relance de la politique sociale européenne », Formation professionnelle, $\mathrm{n}^{\circ} 32$ (May-August 2004): 61-69.

Lapeyre, Jean, Le dialogue social européen. Histoire d'une innovation sociale (1985-2003), ETUI: Bruxelles, 2017.

Leboutte, René, Histoire économique et sociale de la construction européenne, Bruxelles: Peter Lang, 2008.

Meadows, Donella, Meadows, Dennis, Randers, Jorgen and Behrens, William, The Limits to Growth, New York: Universe Books, 1972.

Mechi, Lorenzo, "Du Bureau international du Travail à la politique sociale européenne : les origines d'un modèle », Le Mouvement Social 244, n³ (July-September 2013): 17-30.

Mechi, Lorenzo e Varsori, Antonio, Lionello Levi Sandri e la politica sociale europea, Milano: Angeli, 2008.

Meynaud, Jean Meynaud et Sidjanski, Dusan, Les groupes de pression dans la Communauté européenne, Montréal: Université de Montréal, 1969.

Miard-Delacroix, Hélène, Willy Brandt, Paris: Fayard, 2013.

Muet, Pierre-Alain, Le chômage persistant en Europe, Paris: Presses de la FNSP, 1994.

Olivi, Bino, L'Europe difficile, Paris: Gallimard, 2001.

Paulus, Daniel, La création du Comité permanent de l'emploi des Communautés européennes, Bruxelles: Bruylant, 1972.

Shore, Chris, Building Europe, London: Routledge, 1999.

Spinelli, Altiero, The Eurocrats: Conflict and Crisis in the European Community, Baltimore: The Johns Hopkins Press, 1966.

Varsori, Antonio, Inside the European Community. Actors and Policies in the European Integration 1957-1972, Baden-Baden: Nomos Verlag, 2006.

Varsori, Antonio, "Alle origini di un modello sociale europeo: la Comunità europea e la nascita di una politica sociale (1969-1974)", Ventunesimo Secolo (March 2006): 17-47.

Vayssière, Bertrand, Européiste et eurocrate: la vie fédéraliste de Raymond Rifflet. Toulouse: Presses universitaires du Midi, 2018.

Walsh, John, Patrick Hillery: the Official Biography, Dublin: New Island, 2008.

Wilkens, Andreas Wilkens, Willy Brandt et l'unité de l'Europe. De l'objectif de la paix aux solidarités nécessaires, Bruxelles: Peter Lang, 2011. 